\title{
OBTENCIÓN NO INVASIVA DE LA ONDA DE PRESIÓN ARTERIAL EN LA CARÓTIDA
}

\author{
D. Zambrana-Vinaroz, V. Esteve*, J.M. Vicente, J.M. Sabater-Navarro \\ Universidad Miguel Hernández, Elche-España, dzambrana@umh.es \\ *Universidad de Alicante, Dpto. de Lenguaje y Sistemas Informáticos.
}

\begin{abstract}
Resumen
La monitorización de la onda de presión arterial permite obtener información sobre el funcionamiento cardiovascular del paciente. Por ello, en este artículo se propone un dispositivo no invasivo capaz de captar las vibraciones (ondas de presión) producidas por la arteria carótida mediante un sensor de presión encapsulado en una cúpula cerrada rellena de aire. Con dicho dispositivo es posible realizar un rápido diagnóstico del sistema circulatorio, comprobando el correcto funcionamiento de las válvulas aórticas, gracias a que se puede visualizar el punto dícroto (el punto que discrimina el paso de sístole a diástole). A lo largo del artículo se describen los materiales $y$ métodos que se han usado para llevar a cabo el dispositivo, así como los resultados obtenidos en los que se puede visualizar la morfología de la onda de presión.
\end{abstract}

Palabras clave: Presión arterial, transductor mecánico, cúpula, carótida, punto dícroto, sensor de presión.

\section{INTRODUCCIÓN}

La presión arterial es uno de los principales factores mortales cardiovasculares, la Organización Mundial de la Salud (OMS) concluyó que la hipertensión arterial es la causa más común atribuible a muerte prevenible en los países desarrollados [6]. Dicha enfermedad afecta aproximadamente a uno de cada cuatro adultos y reduce la esperanzada de vida entre 10 y 15 años [1] [3]. A lo largo de la historia se ha intentado monitorizar la presión arterial mediante diferentes métodos.

Stephen Halls descubrió la presión arterial por primera vez en 1733. Usó un tubo de vidrio largo para canalizar la arteria de un caballo y medir la presión. En ese momento, sólo se podían observar pulsaciones arteriales. No fue hasta 1949 cuando la medición intraarterial continua de la presión arterial a través de la canulación se utilizó en entornos clínicos [5].
En 1847, Ludwig desarrolló el quimógrafo, que fue un gran avance para obtener un registro continuo de la presión arterial y otros datos hemodinámicos [10].

Más tarde los investigadores se centraron en el desarrollo de otras técnicas para la monitorización continua de la presión arterial. Así surgieron dos grandes técnicas, la tonometría arterial y el método de pinzamiento de volumen, fueron iniciado por Pressman [14] y Penaz [13]. Sin embargo, estas dos técnicas son invasivas porque requieren la aplicación de fuerza externa y un manguito oclusivo.

Actualmente se usa el esfigmomanómetro o tensiómetro, que es un instrumento médico empleado para la medición indirecta de presión arterial, proporcionando, por lo general, la medición en milímetros de mercurio $(\mathrm{mmHg})$. Se compone de un sistema de brazalete inflable y un manómetro [7]. Sin embargo, en el ámbito clínico, cuando el paciente se encuentra inestable y con tratamientos vasoactivos está indicado canalizar una vía arterial y medir la presión arterial de forma directa ya que esta forma se obtiene datos de forma continua y más precisa que con la medición indirecta (esfigmomanómetro) [12].

Algunos de los métodos y sistemas disponibles en el mercado para el análisis del contorno de la onda de pulso son: $\quad \mathrm{PiCCO}^{\circledR}($ Pulsion) [11], PulseCO ${ }^{\circledR}$ (LiDCO) [9], MostCare ${ }^{\circledR}$ (Vygon) [15].

Además, para analizar la forma de onda de la presión arterial también se usa la fotopletismografía (PPG), que es una técnica óptica simple utilizada para detectar cambios volumétricos en la sangre en la circulación periférica. Dicha técnica se basa en la propiedad de que la sangre absorbe más luz infrarroja que el resto de los tejidos. Esta señal contiene información importante sobre la variabilidad de la frecuencia cardíaca, la presión arterial, la respiración, etc. Dichas señales son de gran importancia en aplicaciones clínicas, ya que pueden ser utilizadas para detectar varios trastornos biológicos como obstrucciones arteriales, enfermedades cardíacas, etc [2].

En la actualidad se están desarrollando dispositivos no invasivos (smartwatch) [8]. Aunque estos realizan una 
estimación de la presión arterial, todavía se están mejorando para aumentar la fiabilidad y precisión de los valores que registra. Además, no son capaces de obtener la onda de presión arterial.

En este artículo se propone un dispositivo capaz de registrar la onda de presión arterial de forma no invasiva mediante un transductor mecánico.

A continuación, en el apartado de metodología se va a proceder a describir las características del dispositivo que se ha desarrollado, los materiales empleados y el funcionamiento y la adquisición de los datos de la onda de presión. Después se mostrarán las conclusiones y resultados.

\section{METODOLOGÍA}

En este apartado se describirán los métodos que se han usado para diseñar el dispositivo capaz de captar las vibraciones producidas por la arteria carótida.

\subsection{SENSOR ANALÓGICO}

Al colocar el dispositivo en la zona de la arteria carótida, las vibraciones de la misma ejercerán una deformación en la cúpula que a su vez llevará a cabo un aumento de presión del aire interior que contiene esta, captando dicho incremento el sensor de presión que se encuentra dentro. Con dichas vibraciones es posible obtener una resolución adecuada de los puntos característicos de dicha onda al igual que se obtienen con dispositivos invasivos. Por tanto, el encargado de registrar los cambios de presión dentro de la cúpula cerrada es el sensor de presión (Figura 1).

En un primer momento se usó un sensor de presión digital, en concreto el modelo BMP280 de la marca Bosch. Los principales motivos por los cuales se escogió este modelo fue el pequeño tamaño que ocupa el sensor $(2.5 \times 2.5 \mathrm{~mm})$ y la capacidad para registrar pequeños cambios de presión. Además, tenía la ventaja de que tenía incorporado diferentes protocolos de comunicación (I2C y SPI) que hacían muy fácil llevar a cabo la lectura de los valores de presión mediante un microcontrolador. A pesar de todas las idóneas características descritas, la frecuencia de muestreo máxima era muy baja (menor de $30 \mathrm{~Hz}$ ) para esta aplicación y no se obtenía una resolución lo suficientemente adecuada para discernir los diferentes puntos clave en una onda de presión arterial.

Por ello se optó por escoger un sensor analógico (Figura 1), en concreto el modelo MPXA6115A6U de la marca NXP, el cual proporciona una tensión en función de la presión medida. También tenía una buena sensibilidad $(45 \mathrm{mV} / \mathrm{kPa})$, pero la principal desventaja que poseía dicho sensor era su tamaño
$(10.5 \times 18 \mathrm{~mm})$. A pesar de dicha desventaja se obtenía una resolución adecuada para discernir los diferentes puntos clave en una onda de presión arterial, principal objetivo del presente artículo. En la Figura 1 se puede observar la placa de circuito impreso (PCB) que se diseñó para poder adquirir la señal de presión con el sensor analógico.

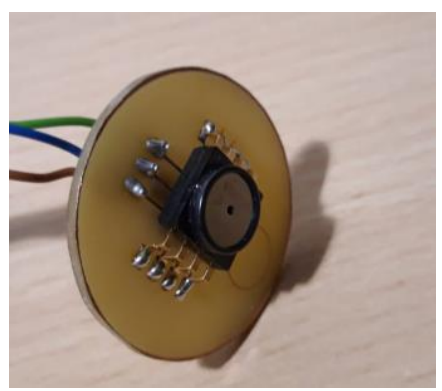

Figura 1:PCB con el sensor de presión analógico.

\subsection{ARDUINO}

Para llevar a cabo la lectura de los valores de presión registrados por el sensor analógico se utilizó el sistema Arduino. Para ello se usó uno de los pines analógicos y se realizó un programa para Arduino (.ino), estableciendo la frecuencia de muestreo del sensor analógico en 4 milisegundos $(250 \mathrm{~Hz})$. Posteriormente, dichos valores de presión eran almacenados para su posterior tratamiento y análisis. En la figura 2 se puede apreciar el código empleado para la adquisición de la señal de presión mediante la placa de Arduino.

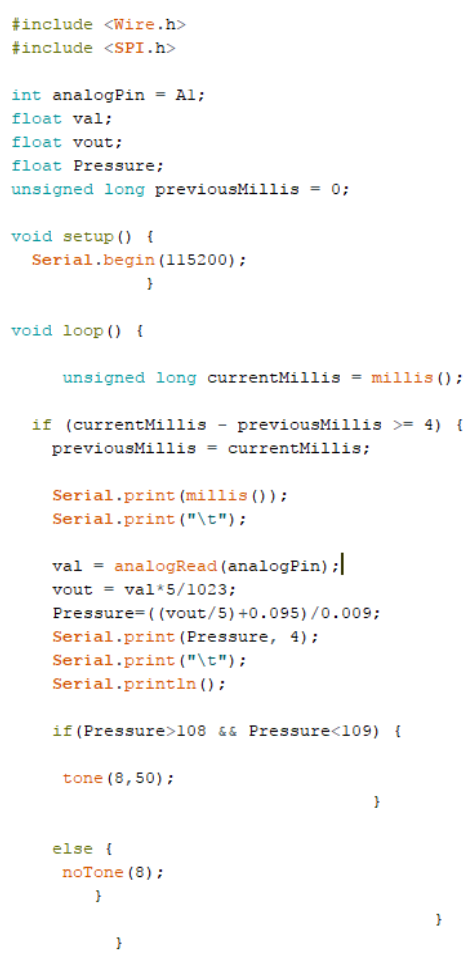

Figura 2: Código Arduino (.ino) 


\subsection{DISEÑO DE LA CÚPULA}

La función que tiene la cúpula es de actuar como transductor mecánico, convirtiendo las vibraciones mecánicas de la arteria en un incremento de presión dentro de la misma.

A la hora de realizar el diseño de la cúpula se usó una lámina de polipropileno. Para darle la forma de cúpula se diseñó una pieza que tuviera un agujero con un diámetro ligeramente menor que el tamaño de la PCB realizada. Posteriormente, con la lámina estirada de polipropileno encima del agujero de la pieza se procedió a ejercer una suave presión con otra pieza (en forma de cúpula) previamente calentada para ir deformando el polipropileno e ir imprimiendo la forma de cúpula deseada (Figura 3 ).

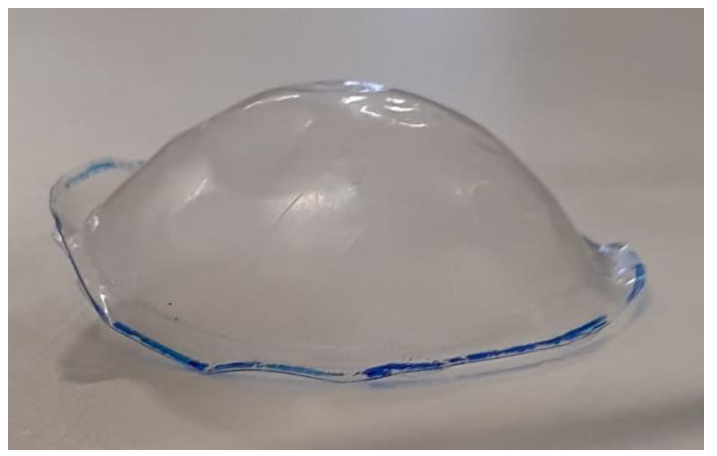

Figura 3: Cúpula.

Finalmente se pegó la cúpula a la PCB mediante un epoxi (figura 4). Cabe destacar que para que el dispositivo funcionase correctamente, era importante sellar muy bien con el epoxi la junta entre la cúpula y la PCB con el objetivo de que no se produjesen fugas. Ya que en el caso de que hubiese alguna fuga no aumentaría la presión dentro de la cúpula cuando se producen las vibraciones de arteria carótida.

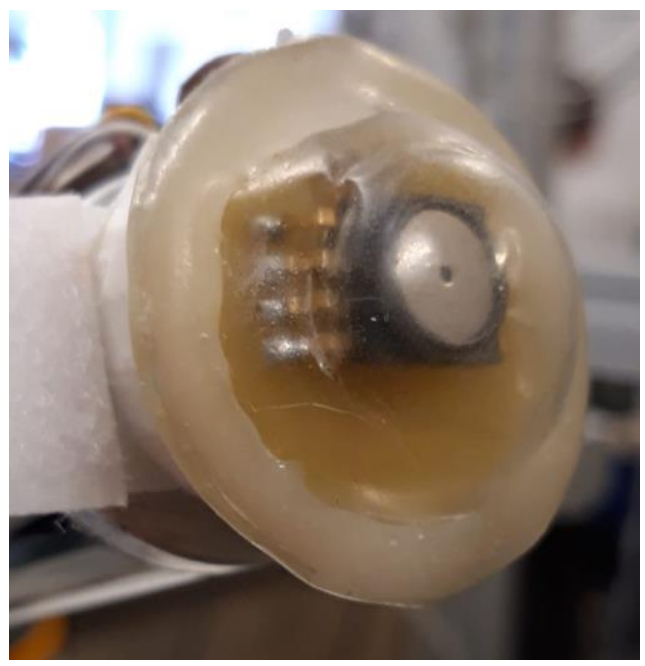

Figura 4: Unión de la PCB con la cúpula.

\subsection{ESTABILIZACIÓN DE LA ONDA}

Debido a que estamos obteniendo los valores de presión en el interior de la cúpula y está última se encuentra sellada, los valores de presión aumentarán al ejercer una fuerza con la mano a la hora de colocar o sostener el dispositivo para realizar las medidas. Es por ello, que a la hora de acoplar el dispositivo diseñado a la zona del cuello donde se encuentra la carótida, se realizó un sistema de soporte fijo anclado a la mesa en cuyo extremo se encuentra el dispositivo de medición de la onda de presión (Figura 5). El objetivo de dicho soporte es que el usuario sea el que se mueva y se acomode el dispositivo, quedando este último fijo para así evitar lo máximo las fluctuaciones de la presión al sostenerlo con la mano. Es destacable que a la hora de adquirir una buena señal es muy importante la colocación del dispositivo, y la calidad de dicha señal dependerá de ello.

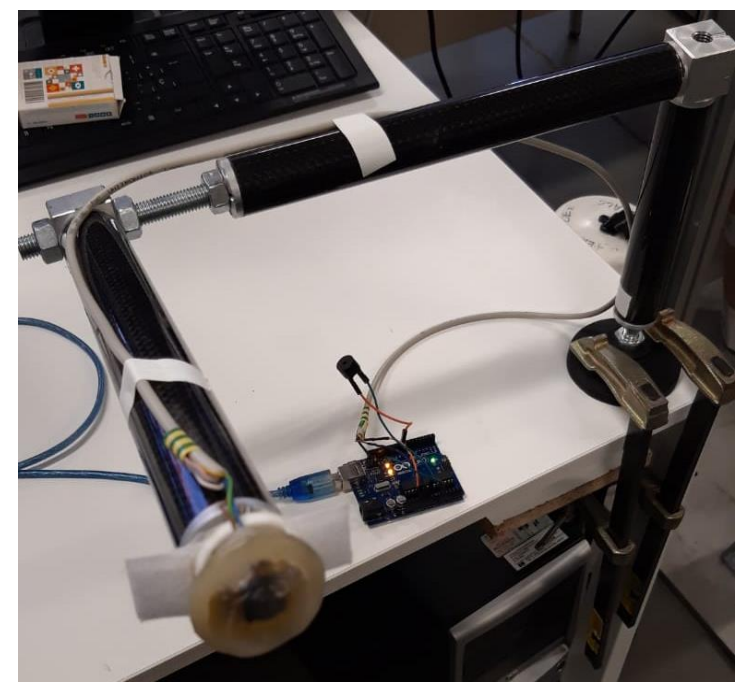

Figura 5: Soporte fijo con el dispositivo de medición en el extremo.

Además, para reducir aún más dichas fluctuaciones se implementó un sistema de aviso mediante un zumbador (Figura 6), el cual avisa cuando las presiones que registra el sensor se encuentran dentro de un rango muy acotado de $108 \mathrm{kPa}$ a $109 \mathrm{kPa}$ $(810,06 \mathrm{mmHg}-817,56 \mathrm{mmHg})$. De esta forma se asegura controlar que la fuerza que ejerce el usuario contra el dispositivo que se encuentra en el extremo del soporte se mantiene constante. Por lo tanto, cuando se están realizando correctamente las medidas, suena un pitido del zumbador.

Para llevar a cabo el sistema de aviso descrito anteriormente se usó uno de los pines digitales de la placa Arduino, activando dicho pin cuando el rango de presión es de 108-109 $\mathrm{kPa}$. En la figura 2 se puede apreciar el código empleado. 


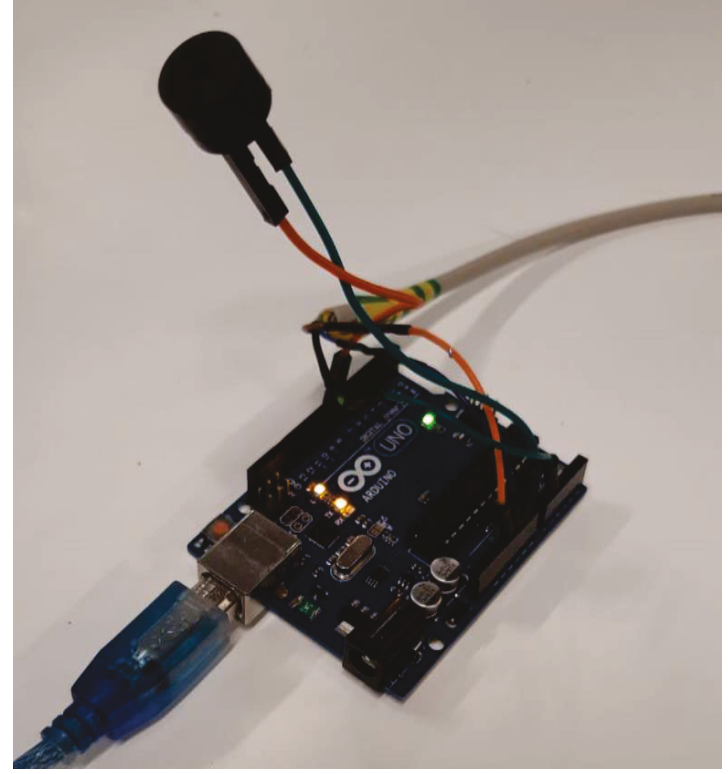

Figura 6: Zumbador avisador.

Con ambas implementaciones se reducen las fluctuaciones de presión y se consigue una mayor estabilidad en la onda de presión tal y como se aprecia en la figura 7. Como consecuencia, esto se traduce en una mejora de la calidad de la señal consiguiendo distinguir los diferentes puntos clave en la onda de presión arterial.

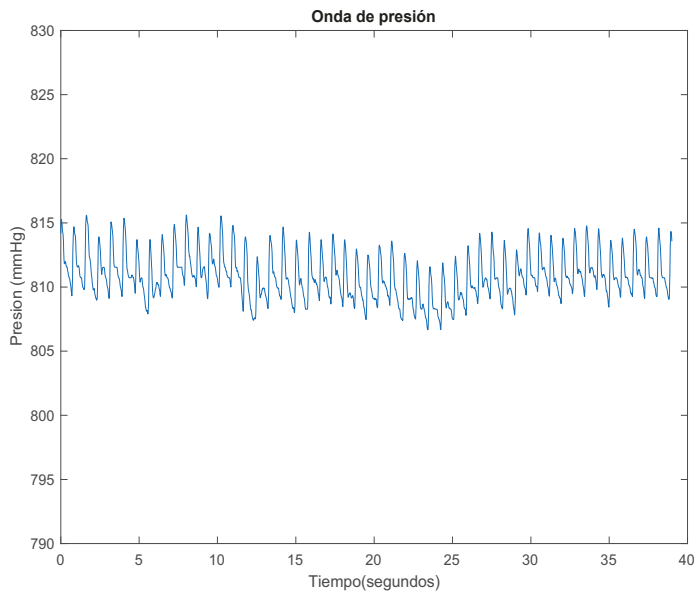

Figura 7: Onda de presión estabilizada.

\subsection{TRATAMIENTO DE LA SEÑAL}

Una vez que se obtienen los valores de presión mediante la placa Arduino, se cargan dichos valores en Matlab. Con objeto de mejorar la onda obtenida y eliminar el ruido que contiene, se realiza un procesamiento que consiste en un suavizado y un filtrado de la señal de presión mediante un filtro de paso bajo con una frecuencia de corte de $10 \mathrm{~Hz}$. En la figura 8 se puede visionar en rojo la onda de presión sin tratar y en azul la onda de presión tras sufrir un suavizado y filtrado.

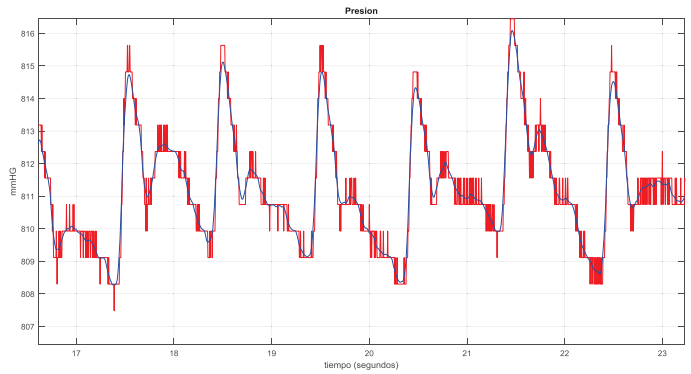

Figura 8: Onda de presión sin tratar en rojo y la onda suavizada y filtrada en azul.

\subsection{CÁlCUlo de PUNTOS ClAVE}

La onda de presión arterial obtenida tiene puntos clave que se corresponden con las fases del ciclo cardíaco (sístole y diástole). La sístole es la fase de contracción del corazón, donde la sangre es bombeada a los vasos sanguíneos, y la diástole es la fase de relajación, que permite que la sangre entre en el corazón.

La presión máxima se obtiene durante el periodo de la eyección ventricular, es decir, en la fase sistólica y la presión mínima se obtiene en el momento final de la diástole, previo a la contracción ventricular. El punto dícroto es el punto que discrimina el paso de sístole a diástole, dicho punto corresponde a un aumento transitorio de la presión aórtica al cerrar la válvula aórtica [4].

Dada la importancia de dichos puntos a la hora de analizar una onda de presión arterial, se realizó un algoritmo para identificar los puntos diastólicos (mínimos de la señal de presión), los puntos sistólicos (máximos de la señal de presión) y las muescas dicróticas (aumento transitorio de la presión aórtica). Dicho algoritmo se basa en detectar los picos de la segunda derivada de la señal (aceleración de la onda) para obtener así los puntos en los que la señal cambia bruscamente y a partir de estos calcular los puntos máximos, mínimos y dícrotos. En la figura 9 se pueden visualizar en color verde los puntos diastólicos, en negro los puntos sistólicos y en magenta los puntos dicróticos.

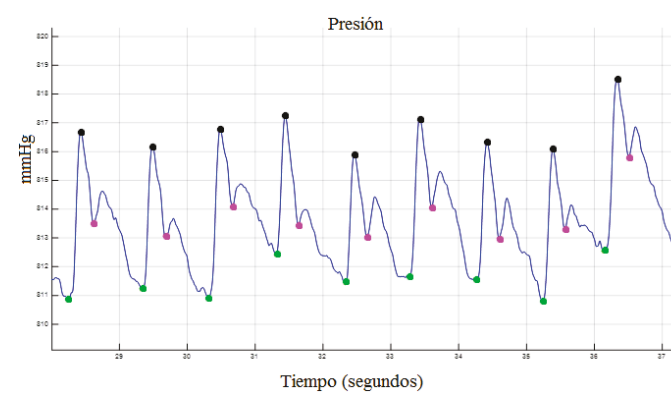

Figura 9: Puntos clave de la onda de presión arterial. 


\section{RESULTADOS}

En este apartado se va a realizar un análisis de los resultados obtenidos, y las distintas líneas futuras de investigación.

\subsection{CONCLUSIONES}

Como se ha ido desarrollando en el epígrafe anterior, se ha logrado con éxito la obtención de la onda de presión en la carótida. La morfología de dicha señal es la que se puede apreciar en la figura 10. En ella se pueden discernir los puntos clave descritos en el punto 2.5. Con dicha onda se puede hacer un rápido diagnóstico del sistema circulatorio, ya que con el envejecimiento, y en numerosas alteraciones arteriales, la rigidez arterial aumenta, provocando importantes cambios en la morfología de la onda de presión arterial [16].

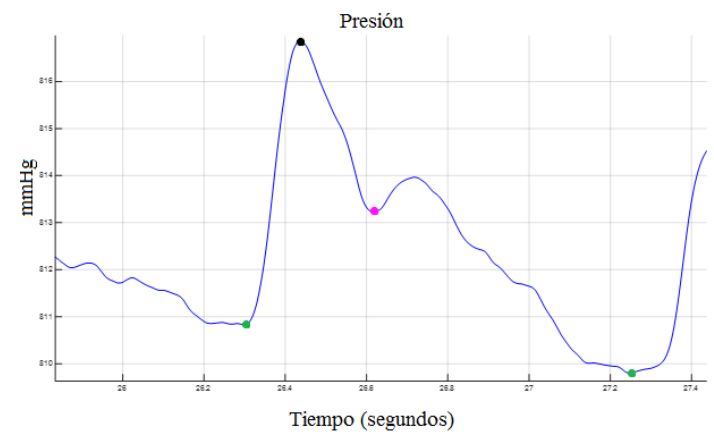

Figura 10: Morfología onda de presión arterial no invasiva.

Si se compara con la señal de presión arterial que se registra de forma invasiva mediante un catéter intraarterial conectado a un transductor de presión (figura 11), se puede observar su similitud de dichas ondas. En ambas se puede visualizar los puntos característicos de forma clara, la única diferencia que existe entre ambas ondas es que con la onda de presión intraarterial se puede saber con precisión la presión arterial sistólica y diastólica (máxima y mínima), lo que hace que sea fácilmente monitorizable en un ámbito clínico.

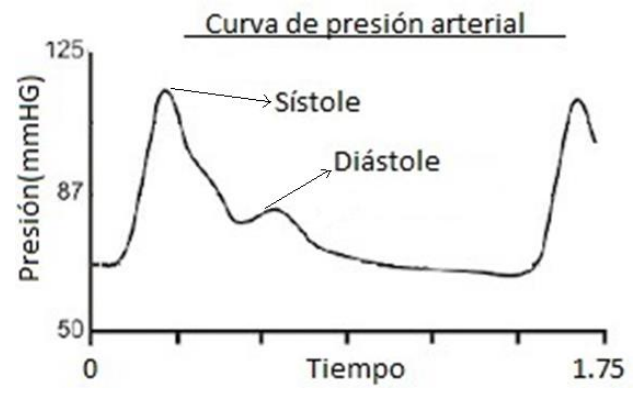

Figura 11: Morfología onda de presión arterial invasiva.

\subsection{LÍNEAS FUTURAS}

Con la obtención de la onda de presión arterial mediante el dispositivo se puede llegar a realizar un diagnóstico del sistema circulatorio. Por ello se espera seguir avanzando en este campo y que el dispositivo descrito en el presente artículo sirva de base para poder llegar a realizar una monitorización y estimación de los valores de presión arterial (presión sistólica y diastólica).

\section{Agradecimientos}

Los autores quieren agradecer el apoyo económico a la EPSE-UMH, al proyecto "Craneeal" - Referencia: DPI2016-80391-C3-2-R [AEI/FEDER, UE], financiado por la Agencia Estatal de Investigación (AEI) y por la Unión Europea a través del Fondo Europeo de Desarrollo Regional - FEDER - "Una manera de hacer Europa". D. Zambrana agradece el apoyo económico del programa ACIF con la beca ACIF/2019/058.

\section{English summary}

\section{NON-INVASIVE OBTENTION OF THE BLOOD PRESSURE WAVE IN THE CAROTID ARTERY}

\begin{abstract}
Blood pressure wave monitoring provides information about the patient's cardiovascular function. For this reason, this article proposes a non-invasive device capable of capturing the vibrations (pressure waves) produced by the carotid artery by means of a pressure sensor encapsulated in a closed dome filled with air. With this device it is possible to carry out a quick diagnosis of the circulatory system, checking the correct functioning of the aortic valves, thanks to the fact that the dicrotic notch (the point that discriminates the passage from systole to diastole) can be visualised. Throughout the article, the materials and methods used to carry out the device are described, as well as the results obtained in which the morphology of the pressure wave can be visualized.
\end{abstract}

Keywords: Blood pressure, mechanical transducer, dome, carotid, dicrotic notch, pressure sensor.

\section{Referencias}

[1] (2013) "Información general sobre la HIPERTENSIÓN en el mundo. Día mundial de la Salud.", Organización Mundial de la Salud 
http://apps.who.int/iris/bitstream/10665/87679/1/WH O DCO WHD 2013.2 spa.pdf?ua=1.

[2] Allen J., (2007) "Photoplethysmography and its application in clinical physiological measurement," Physiological Measurement, pp. R1-R39.

[3] Bancalari R., Díaz C., Martínez-Aguayo A., et al. (2011) "Prevalencia de hipertensión arterial y su asociación con la obesidad en edad pediátrica". Rev Med Chil., 139, pp. 872-879.

[4] Cymberknop L., Legnani, W., Pessana, F., Armentano, R., (2013) "Procesamiento no lineal de señales cardiovasculares: marco conceptual para la detección de patologías". Proyecciones. pp 11-28.

[5] Ding X.,(2016) "Continuous Blood Pressure Measurement From Invasive to Unobtrusive: Celebration of 200th Birth Anniversary of Carl Ludwig," IEEE Journal of Biomedical and Health Informatics, pp. 1455-1465.

[6] Ezzai M., López AD., Rodgers A., Van der Hoorn S., Murray CJ., (2002). "Comparative Risk Assesment Collaborating Group. Selected major risk factors and global and regional burden of disease". Lancet; 360, pp. 1347-60.

[7] Gonzalez M., (2018) "Análisis de concordancia entre un esfigmomanómetro manual y otro digital usados en una unidad militar, en reposo y tras un ejercicio físico". Sanid. Mil., pp.13-19.

[8] Georgi N., Corvol A., Jeannès R., (2018), "For a More Reliable Measure of Wrist Blood Pressure Using Smartwatch". Telemedicine and e-Health. https://doi.org/10.1089/tmj.2018.0112

[9] Hamilton T.T., Huber L.M., Jessen M.E. (2002) "PulseCO: a less-invasive method to monitor cardiac output from arterial pressure after cardiac surgery". Ann Thorac Surg. pp.1408-12.

[10] Ludwig C, (1847) “Contribution to Knowledge of the respiratory movements influence to the aortic system circulation" Beitr"age zur Kenntnis des Einflusses der Respirationsbewegungen auf den Blutlauf im Aortensysteme, pp. 242-302.

[11] Martín A., Saboya S., Patiño M., Silva J.A., Gómez S., Blanco J.J., (2008) "Monitorización hemodinámica: Sistema PiCCO®", Enfermería intensiva, pp.134-140.

[12] Mateu A., Ferrándiz G., Gruartmoner J., Mesquida C., Sabatier Y., (2012) "Técnicas disponibles de monitorización hemodinámica. Ventajas y limitaciones." Med Intensiva. 36 pp 434444.

[13] Penaz J., (1973) "Photoelectric measurement of blood pressure, volume and flow in the finger," Proc. Dig. 10th Int. Conf. Med. Biol. Eng., pp. 2-7.

[14] Pressman G., Newgard P., (1963) "A transducer for the continuous external measurement of arterial blood pressure," IEEE Trans. Bio-Med. Electron, pp. 73-81.

[15] Romagnoli S., Bevilacqua S., Lazzeri C., Ciappi F, Dini D., Pratesi C., Gensini GF., Romano SM. (2009) "Most care ${ }^{\circledR}$ : a minimally invasive system for hemodynamic monitoring powered by the pressure recording analytical method (PRAM)" HSR Proc Intensive Care Cardiovasc Anesth. pp.20-27.

[16] Zocalo Y., (2012) "Presión aórtica central y parámetros de reflexión de onda: Importancia clínica y análisis de consistencias y discrepancias con niveles de presión periférica". Rev.Urug. Cardiol, pp.418-430.

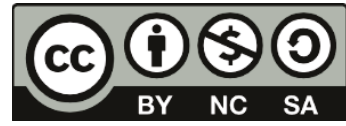
(C) 2019 by the authors. Submitted for possible open access publication under the terms and conditions of the Creative Commons Attribution CC BY-NC-SA 4.0 license (https://creativecommons.org/licenses/bync-sa/4.0/deed.es). 